\title{
Thermal and Electric Cloaking Effect in Concentric Composite Made of Homogeneous Bulk and Porous Semiconductors
}

\author{
ROLAND H. TARKHANYAN $\mathbb{1}^{1,2,3}$ and DIMITRIS G. NIARCHOS ${ }^{1}$ \\ 1.-Institute of Nanoscience and Nanotechnology of NCSR "Demokritos", Neapoleos \& Patriar- \\ chou Grigoriou, 15310 Athens, Greece. 2.-Institute of Radiophysics \& Electronics of NAS of \\ Armenia, 0203 Ashtarak, Armenia. 3.-e-mail: rolandtarkhanyan@yahoo.com
}

\begin{abstract}
A new material platform is presented to manipulate heat and charge transportation in steady-state conditions. More precisely, we investigate the conceptual realization of a concentric composite made of the same isotropic and homogeneous semiconductor material with layers of different porosity, and show the possibility of a simultaneous cloaking performance in such a device for both heat flux and electric current without disturbing external fields. The background medium in the composite is a porous material with a periodical $3 \mathrm{D}$ cubic lattice of spherical hollow pores while the cylindrical shell is made from the same bulk material with zero porosity. A sound analytical expression is found for the volume fraction of the pores at which bi-functional cloaking effect can be realized. To validate our theoretical results, we also demonstrate the temperature and heat flux profiles as well as the voltage and current profiles in numerical simulations for a composite consisting of bulk (cylindrical shell) and porous (background) $n$-type silicon layers.
\end{abstract}

Key words: Thermoelectricity, semiconducting composite, conductivity, porosity, cloaking

One of the most exotic and up-to-date applications of transformation electrodynamics and thermodynamics consists in the possibility of electric current cloaking and heat manipulation using thermal cloaks and heat concentrators. ${ }^{1-8}$ In recent work, ${ }^{9}$ it has been reported that bilayer copper and polystyrene cylinders can cloak steady current, so as electric potential gradients are parallel and equal outside the outer cylinder while the electric potential is zero inside the inner cylinder. Using transformation optics methods, ${ }^{10-12}$ a metamaterial construction has been designed which simultaneously behaves as a thermal concentrator and an electrical invisibility cloak. ${ }^{13}$ Various thermal cloaking devices have been experimentally demonstrated in Ref. 14-17 The authors of Ref. 18 showed that a two-dimensional circular thermal cloak can be achieved using layers with homogeneous and constant thermal conductivities. Obviously, such

(Received June 8, 2015; accepted September 9, 2015; published online September 23, 2015) devices can open new perspectives for applications based on the manipulation of heat and electrical currents. The purpose of this paper is an investigation of the following problem: is it possible to have a simultaneous cloaking performance for both heat flux and electric current in a composite made of the same isotropic and homogeneous semiconducting material with layers of different porosity? We have to note that porous semiconductors are of great interest for their potential in converting heat into electricity due to lower thermal conductivity and higher thermoelectric figure of merit. ${ }^{19-21}$ Our calculations confirm the possibility of bi-functional cloaking effect in a cylindrical composite of bulk and porous layers at a definite volume fraction of the pores to satisfy dual cloaking conditions. The question of interest here is the possibility to realize coupling of different physical phenomena in a single device to better manipulate the electric current and heat flux simultaneously. Undoubtedly, this bicloaking effect can find many potential applications, such as technology of motors, generators, inductors, and engines, as well as electric impedance tomography, natural resource exploration and military concealment. 
Consider a composite (Fig. 1) consisting of background medium (porous semiconductor) with electric and thermal conductivities $\sigma_{1}$ and $\kappa_{1}$ (region 1) and a cylindrical shell (region 2, bulk semiconductor, conductivities: $\sigma_{2}, \kappa_{2}, 2 \mathrm{D}$ cross-section is a strip between two concentric circles of radius $R_{1}$ and $R_{2}, R_{2}>R_{1}$ ). Cross-section of region 3 (a vacuum or an isolating material with $\kappa_{3}<<\kappa_{1,2}$ and $\sigma_{3}<<\sigma_{1,2}$ ) is a cylindrical shell with external radius $R_{1}$ and inner one $R_{0}$. Region 4 with radius $r<R_{0}$ is a full cylinder (cloak subject) with arbitrary conductivities $\sigma_{4}$ and $\kappa_{4}$.

In an isotropic homogeneous medium, the continuity equation for heat flux density $\mathbf{W}=-\kappa \nabla T$ ( $\kappa$ is thermal conductivity, $\nabla T$ is the temperature gradient) is given by the Laplace equation

$$
\nabla^{2} T=0 .
$$

The continuity equation for the electric current density $\mathbf{J}=\sigma \mathbf{E}=-\sigma \nabla V$ has an analogical form: $\nabla^{2} V=0$. Let a constant temperature gradient $\nabla T$ (or an electric field $E_{0}$ ) be applied along the $x$-axis in Cartesian coordinate system and the cylinder axis along the $z$-axis. After the system reaches thermal equilibrium, the temperature and electrical potential in the cylindrical coordinate system $(r, \theta, z)$ in the four above-mentioned regions of the structure can be written in the form

$$
\begin{aligned}
T_{1} & =\left(-r \nabla T+A_{1} / r\right) \cos \theta, T_{2,3} \\
& =\left(B_{2,3} r+A_{2,3} / r\right) \cos \theta, T_{4}=B_{4} r \cos \theta ; \\
V_{1}= & \left(-E_{0} r+A_{1}^{\prime} / r\right) \cos \theta, V_{2,3} \\
= & \left(B_{2,3}^{\prime} r+A_{2,3}^{\prime} / r\right) \cos \theta, V_{4}=B_{4}^{\prime} r \cos \theta .
\end{aligned}
$$

Note that the origins of both the $2 \mathrm{D}$ rectangular and polar coordinate systems are chosen in the center of region 4 . In the case when the temperature gradient is applied to a homogeneous material, the temperature is given by a linear function $T(x)=T_{0}-x \nabla T$, where $T_{0}=\left(T_{\mathrm{h}}+T_{\mathrm{c}}\right) / 2, \nabla T=\left(T_{\mathrm{h}}-T_{\mathrm{c}}\right) / L, T_{\mathrm{h}}, T_{\mathrm{c}}$ represent the temperatures of the hot and cold sides in Fig. 1 , and $L$ is the distance between them. In Eq. 2, for the null point of the temperature scale, the temperature $T_{0}$ at the plane $x=0$ is chosen. By analogy, for the null point of the electric potential scale in Eq. 3, the potential $V_{0}=[V(L / 2)-$ $V(-L / 2)] / 2$ is chosen.

Heat flux and electric current densities in the polar coordinate system are given by

$$
\begin{aligned}
& \mathbf{W}=-\kappa\left(\frac{\partial T}{\partial r} \mathbf{e}_{\mathbf{r}}+\frac{1}{r} \frac{\partial T}{\partial \theta} \mathbf{e}_{\theta}\right) \\
& \mathbf{J}=-\sigma\left(\frac{\partial V}{\partial r} \mathbf{e}_{\mathbf{r}}+\frac{1}{r} \frac{\partial V}{\partial \theta} \mathbf{e}_{\theta}\right)
\end{aligned}
$$

where $\mathbf{e}_{\mathbf{r}}$ and $\mathbf{e}_{\theta}$ are the unit vectors. Using Eq. 4a for the heat flux density in various regions, one can obtain

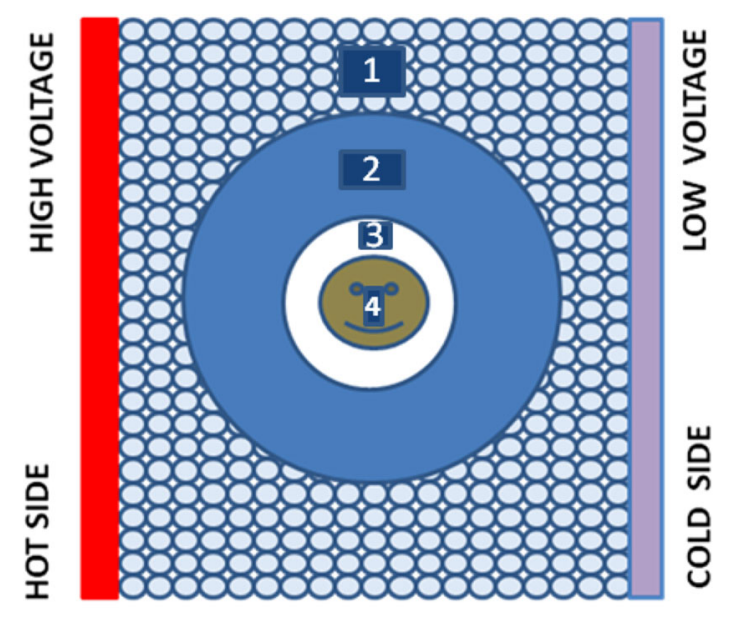

Porous Material $\left(k_{1}, \sigma_{1}\right)$

Bulk Material $\left(k_{2}, \sigma_{2}\right)$

Fig. 1. Schematic illustration of the bi-functional cloaking construction.

$\mathbf{W}_{\mathbf{1}}=\kappa_{1}\left[\left(\nabla T+\frac{A_{1}}{r^{2}}\right) \cos \theta \mathbf{e}_{\mathbf{r}}+\left(-\nabla T+\frac{A_{1}}{r^{2}}\right) \sin \theta \mathbf{e}_{\theta}\right]$,

$$
\begin{aligned}
\mathbf{W}_{\mathbf{2}, 3}= & \kappa_{2,3}\left[\left(-B_{2,3}+\frac{A_{2,3}}{r^{2}}\right) \cos \theta \mathbf{e}_{\mathbf{r}}\right. \\
& \left.+\left(B_{2,3}+\frac{A_{2,3}}{r^{2}}\right) \sin \theta \mathbf{e}_{\theta}\right] \\
\mathbf{W}_{\mathbf{4}}= & -\kappa_{4} B_{4}\left(\cos \theta \mathbf{e}_{\mathbf{r}}-\sin \theta \mathbf{e}_{\theta}\right) .
\end{aligned}
$$

Neglecting quantities $\sim \kappa_{3} / \kappa_{2}<<1$ and using Eqs. 2, 5a-5c with standard continuity conditions for the temperature and radial components of the heat flux at the region boundaries, one can find the following analytical expressions for all six unknown coefficients $A_{1,2,3}$ and $B_{2,3,4}$ :

$$
\begin{gathered}
A_{1}=\left(\frac{\gamma \kappa_{2}-\kappa_{1}}{\gamma \kappa_{2}+\kappa_{1}}\right) R_{2}^{2} \nabla T, A_{2}=-\frac{2 R_{1}^{2} R_{2}^{2} \kappa_{1} \nabla T}{\left(R_{1}^{2}+R_{2}^{2}\right)\left(\gamma \kappa_{2}+\kappa_{1}\right)}, \\
A_{3}=\frac{4\left(R_{0} R_{1} R_{2}\right)^{2} \kappa_{1} \nabla T}{\left(R_{1}^{2}-R_{0}^{2}\right)\left(R_{1}^{2}+R_{2}^{2}\right)\left(\kappa_{1}+\gamma \kappa_{2}\right)}, \\
B_{2}=\frac{A_{2}}{R_{1}^{2}}, B_{3}=-\frac{A_{3}}{R_{0}^{2}}, B_{4}=-\frac{2 \kappa_{3} A_{3}}{\kappa_{4} R_{0}^{2}}
\end{gathered}
$$

where

$$
\gamma \equiv \frac{R_{2}^{2}-R_{1}^{2}}{R_{2}^{2}+R_{1}^{2}} .
$$

Expressions for the electric current vectors in the corresponding space regions have the form analo- 
gous to those in Eq. 5a-5c. In the case when $\sigma_{3} / \sigma_{2}<<1$, the coefficients $A_{1,2,3}^{\prime}$ and $B_{2,3,4}^{\prime}$ in Eq. 3 are given by the same Eqs. $6-9$, where $\kappa_{i}(i=1,2,3)$ and $\nabla T$ should be replaced by $\sigma_{i}$ and $E_{0}$, respectively.

Note now that if $\kappa_{3}<<\kappa_{4}$, Eq. 8 gives $B_{4} \approx 0$; it means that heat current in region $4\left(r<R_{0}\right)$ vanishes: $\mathbf{W}_{\mathbf{4}} \simeq 0$. Analogously, $B_{4}^{\prime} \approx 0$ if $\sigma_{3}<<\sigma_{4}$, and then the condition for electrical cloaking performance in region 4 is fulfilled $\left(\mathbf{J}_{4} \simeq 0\right)$.

Note also that in order not to distort the applied temperature gradient or steady electric field in external region 1 , i.e., to achieve $\mathbf{W}_{\mathbf{1}}=$ $\left\{-\kappa_{1} \nabla T, 0,0\right\}$ and $\mathbf{J}_{1}=\left\{\sigma_{1} E_{0}, 0,0\right\}$ in Cartesian coordinate system, the conditions

$$
A_{1}=0, A_{1}^{\prime}=0
$$

must be required. Then, from Eqs. 6 and 10, one can find the dual cloaking condition in the form

$$
\frac{\kappa_{1}}{\kappa_{2}}=\frac{\sigma_{1}}{\sigma_{2}}=\gamma
$$

To understand the peculiarities of the proposed design, it is useful to consider it in terms of transformation technique. Let us imagine a virtual space region in a cylindrical coordinate system $\left(r^{\prime}, \varphi^{\prime}, z^{\prime}\right)$ filled by porous medium with conductivities $k_{1}$ and $\sigma_{1}$. Then, consider a transformation where outside the radius $R_{2}$ the mapping is identity mapping, while inside the radius $R_{2}$ coordinate transformations are $r=R_{0}+r^{\prime}\left(R_{2}-R_{0}\right) / R_{2}, \varphi=\varphi^{\prime}, \quad z=z^{\prime}$. Thus, the space region outside the origin cylinder with radius $r=R_{2}$ remains undistorted (region 1), while the point of the origin in the virtual $2 \mathrm{D}$ space is expanded into a cavity with radius $r<R_{0}$ in the physical space (region 4). Due to the form invariance, the form of the conduction equations in the distorted coordinates stays unchanged, but the thermal conductivity inside the cloaking shell with $R_{0} \leq r \leq R_{2}$ takes a tensorial form with principal components $k_{r}=k_{1}\left(r-R_{0}\right) / r, \quad k_{\varphi}=k_{1} r /\left(r-R_{0}\right)$, and $k_{z}=k_{r} R_{2}^{2} /\left(R_{2}-R_{0}\right)^{2}$. Thus, the thermal conductivity of the cloaking shell is anisotropic, spatially variable, and also has a singularity at $r=R_{0}$. It is important to note that the radial component $k_{r}$ is always less than tangential component $k_{\varphi}$ and that $0 \leq k_{r} \leq k_{1}\left(1-R_{0} / R_{2}\right)$. Obviously, all these statements are true and analogous for the electrical conductivity tensor components. To exclude the singularity of the cloaking shell, we have used a composite consisting of two homogeneous concentric cylindrical layers with constant thermal conductivities $k_{2}$ and $k_{3}$ (regions 2 and 3 ). High contrast in the conductivity parameter between these regions $\left(k_{3} \ll k_{2}\right)$ provides the necessary anisotropy of the cloaking shell.

For simplicity, as a porous medium, we consider a semiconducting material with a periodical $3 \mathrm{D}$ cubic lattice of spherical hollow pores, while the cylin- drical shell is made from the same bulk material with zero porosity. In Ref. 19 it has been shown that, in a "gray" medium approximation, neglecting free charge carrier contribution in thermal conductivity,

$$
\frac{\kappa_{1}}{\kappa_{2}}=\frac{1-\varphi}{1+3 \varphi / 2 d}
$$

where $\varphi$ is the porosity, is the average phonon mean free path (MFP) in the bulk material and $d$ is the diameter 5 of the pores. Then, from Eqs. 11 and 12 , for the porosity we obtain the value

$$
\varphi=\frac{1-\gamma}{1+3 \gamma / 2 d} .
$$

For estimations, let as assume that $d=30 \mathrm{~nm}$, $R_{0}=0.2 \mathrm{~cm}, R_{1}=0.5 \mathrm{~cm}$ and $R_{2}=1 \mathrm{~cm}$. The phonon MFP in bulk $\mathrm{Si}$ at room temperature $\Lambda=300 \mathrm{~nm}^{22,23}$ Then, from Eqs. 13 and 9, we obtain $\varphi=4 \%$.

In Fig. 2, temperature and heat flux profiles are shown in the cloaking device with $4 \times 4 \mathrm{~cm}$ sizes of the cross-section, at $T_{\mathrm{h}}=100^{\circ} \mathrm{C}, \quad T_{\mathrm{c}}=0^{\circ} \mathrm{C}, \kappa_{2}=$ $147 \mathrm{w} / \mathrm{mK}$ and $\kappa_{1}=88.2 \mathrm{w} / \mathrm{mK}$. We see that in region 4 with $r<R_{0}=0.2 \mathrm{~cm}$ the temperature gradient $\nabla T=0$ and there is no heat flux, while both $\nabla T$ and heat flux in external region 1 are the same as in the absence of the cloaking subject.

Consider now the cloaking conditions when an external static electric field is applied. For the electrical conductivity, we will use a classical expression based on the solution of the Boltzmann transport equation under relaxation time approximation. It can be written in the form

$$
\sigma=\frac{2 q^{2} \sqrt{2 m^{*}}}{3 \pi^{2} \hbar^{3}} G_{3 / 2}\left(\zeta^{*}\right),
$$

where

$$
G_{3 / 2}\left(\zeta^{*}\right)=\int_{0}^{\infty} \varepsilon^{3 / 2}\left(-\partial f_{0} / \partial \varepsilon\right) \tau(\varepsilon) \mathrm{d} \varepsilon,
$$

$f_{0}(\varepsilon, T)=\left(\exp \left[(\varepsilon-\zeta) / k_{B} T\right]+1\right)^{-1}$ is the Fermi distribution function, $m^{*}$ is the effective mass and $\tau(\varepsilon)$ is the combined relaxation time. Using Matthiessen's rule for scattering events which are independent from each other, $\tau(\varepsilon)$ can be expressed $\mathrm{as}^{24,25}$

$$
\tau^{-1}=\tau_{b}^{-1}+\tau_{p}^{-1},
$$

where $\tau_{b}$ is the electronic momentum relaxation time in the bulk matrix and $\tau_{\mathrm{p}}$ is that due to scattering at pore/medium interfaces. To find the relaxation time, let us estimate electron MFP $\Lambda_{\mathrm{el}}$ due to collisions at the pores.

When a charge carrier travels a distance $L$, the average number of its collisions with pores is equal to 


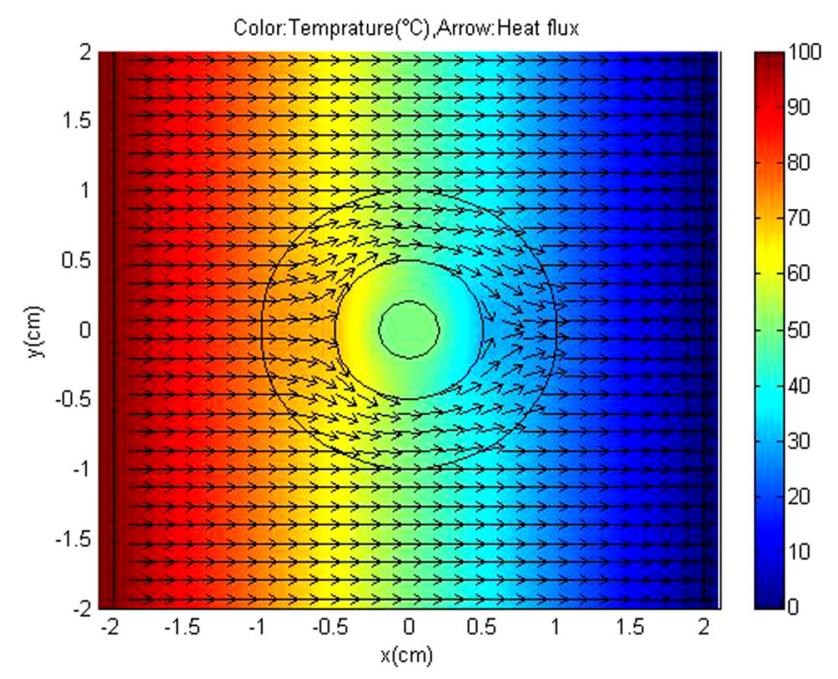

Fig. 2. Temperature (color) and heat flux (arrows) profile at $R_{0}=0.2 \mathrm{~cm}, R_{1}=0.5 \mathrm{~cm}$ and $R_{2}=1 \mathrm{~cm}$ (Color figure online).

$$
\beta=L A c,
$$

where

$$
A=\pi \mathrm{d}^{2} / 4
$$

is the classical cross-section area and $\mathrm{c}$ is the pore number density. The latter can be expressed as a linear function of porosity ${ }^{19}$ :

$$
c=6 \varphi / \pi \mathrm{d}^{3} .
$$

Therefore,

$$
\Lambda_{e l}=L / \beta=(A c)^{-1},
$$

and then $\tau_{p}$ can be found as

$$
\tau_{p}=\frac{\Lambda_{e l}}{v}=\frac{\mathrm{d}\left(2 m^{*}\right)^{1 / 2} \varepsilon^{-1 / 2}}{3 \varphi},
$$

where $v=v(\varepsilon)$ is the electronic velocity. Thus, from Eq. 16, for the combined relaxation time, we obtain

$$
\tau(\varepsilon)=\frac{\tau_{b}}{1+3 \tau_{b}\left(\varepsilon / 2 m^{*}\right)^{1 / 2} \varphi / \mathrm{d}} .
$$

For definiteness, we will further consider the case of relatively low impurity concentrations, so that the main contribution in the carrier relaxation time at zero porosity is from the scattering on deformation potential of acoustic phonons. Then, setting ${ }^{24,25}$

$$
\tau_{b}=a \varepsilon^{-1 / 2},
$$

where

$$
a=\frac{2 \pi \hbar^{4} \rho u^{2}}{k_{B} T\left(2 m^{*}\right)^{3 / 2} D^{2}}
$$

is the constant for acoustic phonons, $\rho$ is the mass density, $u$ the longitudinal velocity of the sound, and

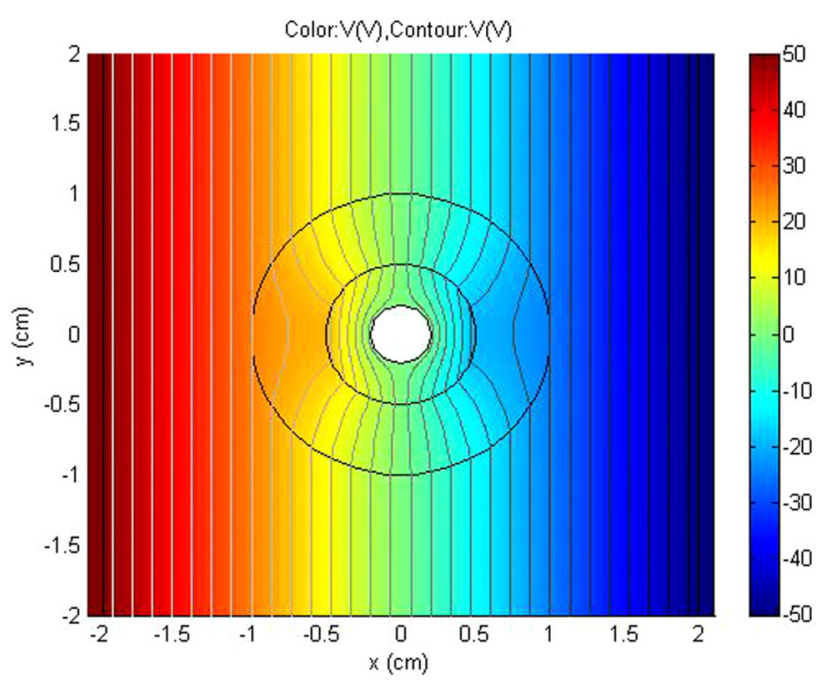

Fig. 3. The numerical voltage distribution. The lines represent isopotential surfaces in cross-section (Color figure online).

$D$ the deformation potential constant, Eq. 22 can be transformed to the form

$$
\tau(\varepsilon)=\frac{a \varepsilon^{-1 / 2}}{1+3 a \varphi / d \sqrt{2 m^{*}}} .
$$

Substituting Eqs. 25 into 14, for the ratio $\sigma_{1} / \sigma_{2}$ in the case of a non-degenerate $n$-type isotropic semiconductor with a parabolic conduction band, we obtain

$$
\frac{\sigma_{1}}{\sigma_{2}}=\frac{1-\varphi}{1+3 \alpha \varphi / \mathrm{d} \sqrt{2 m^{*}}},
$$

where

$$
\sigma_{2}=\frac{n e^{2} \overline{\tau_{b}}}{m^{*}}
$$

is the conductivity of bulk material, $n$ is the carrier density and $\overline{\tau_{b}}$ is the effective relaxation time. When the main contribution in the carrier relaxation time is from the scattering on the deformation potential of acoustic phonons,

$$
\overline{\tau_{b}}=\frac{4 \alpha}{3 \sqrt{\pi k_{B} T}} .
$$

Using Eqs. 13 and 26, one can see that dual cloaking is only possible simultaneously if the following condition is fulfilled:

$$
\alpha\left(2 m^{*}\right)^{-1 / 2}=\Lambda / 2 .
$$

Substituting here $m^{*}=0.26 m_{0}$ for $\mathrm{Si}$, $m_{0}=9.1 \times 10^{-31} \mathrm{~kg}$ and $=300 \mathrm{~nm}$, one can find $\alpha=1.03 \times 10^{-22}\left[\mathrm{~kg}^{1 / 2} \mathrm{~m}\right]$. Assuming that the carrier density $n=1.226 \times 10^{13} \mathrm{~cm}^{-3}$ and using Eqs. 27, 28 , for the conductivity of the bulk material at room temperature we obtain $\sigma_{2}=1.35^{-1} \mathrm{~m}^{-1}$. The voltage 


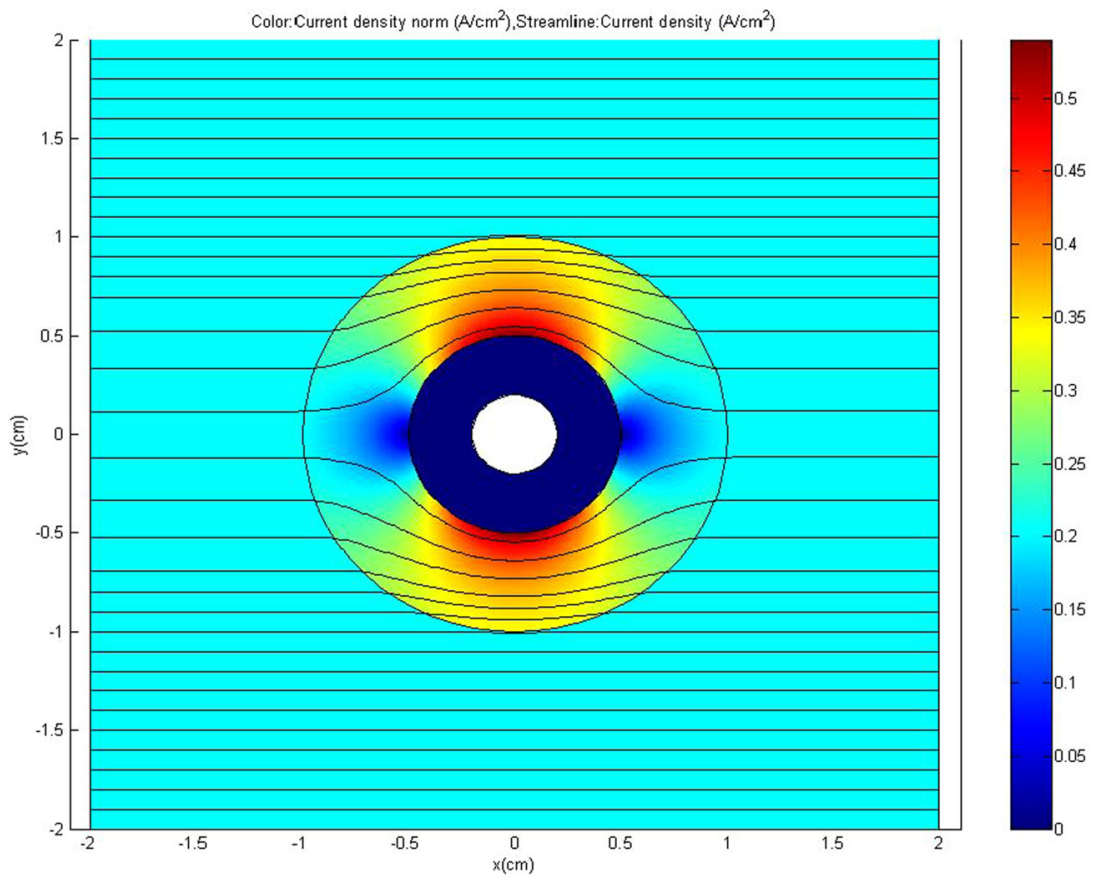

Fig. 4. Current density (color) streamlines at $\sigma_{1}=0.81^{-1} \mathrm{~m}^{-1}$ and $\sigma_{2}=1.35^{-1} \mathrm{~m}^{-1}$ (Color figure online).

distribution and the illustration of current density streamlines at these data are demonstrated in Figs. 3 and 4, respectively. From these figures, one can see the absence of a voltage and also perfect electric current cloaking in space region 4 with radius $r<R_{0}$.

In conclusion, we have presented an analytical design of a bi-functional thermal and electrical cloak based on a multilayer cylindrical composite made of homogeneous, isotropic and non-singular layers with finite constant thermal and electrical conductivities. It should be pointed out that both layers of the proposed coating bi-layer are made from the same semiconducting material of different porosities. We have shown that such a structure behaves as a bi-functional cloak which can provide blocking of both thermal flux and d.c. electric current simultaneously. The tunable thermal $\left(\kappa_{1}\right)$ and electrical $\left(\sigma_{1}\right)$ conductivities of the background medium by adjusting the porosity allow the satisfying of the condition of simultaneously bi-functional cloaking and the controlling of both the heat flux and electrical current such that they pass the cloaking object and return to their original pathway, as if the object was not there.

Assuming that the main contribution in the carrier relaxation time at zero porosity comes from the scattering on the deformation potential of acoustic phonons, we have calculated the ratio $\sigma_{1} / \sigma_{2}$ in the case of a non-degenerate $n$-type isotropic semiconductor with a parabolic conduction band and found a condition at which dual cloaking can be realized simultaneously. As an example, we have examined the cloaking effect in a construction consisting of porous silicon with porosity $\varphi=4 \%$ and electrical conductivity $\sigma_{1}=0.81^{-1} \mathrm{~m}^{-1}$ for background medium and bulk silicon with thermal and electrical conductivities $\kappa_{2}=147 \mathrm{Wm}^{-1} \mathrm{~K}^{-1} \quad$ and $\sigma_{2}=1.35^{-1} \mathrm{~m}^{-1}$ for cylindrical shell of inner radius $R_{1}=0.5 \mathrm{~cm}$ and external radius $R_{2}=1 \mathrm{~cm}$.

It is evident that, due to a lower cost and much easier fabrication, such a device has a strong advantage compared with analogous ones made of meta-materials or anisotropic and inhomogeneous materials, and can find many practical applications to control the electric current and heat propagation simultaneously.

\section{ACKNOWLEDGEMENTS}

The authors would like to acknowledge the financial support of the Project KRIPIS funded by the ESPA program. Also, we thank Nikolaos Dallis for technical help.

\section{REFERENCES}

1. R. Fleury and A. Alu, Forum for electromagnetic research method and applications technologies (FERMAT), 1, (2014).

2. R. Schittny, M. Kadic, S. Guenneau, and M. Wegener, Phys. Rev. Lett. 110, 195901 (2013).

3. M. Kadic, T. Bückmann, R. Schittny, and M. Wegener, Rep. Prog. Phys. 76, 126501 (2013).

4. E. Dede, T. Nomura, P. Schmalenberg, and J.S. Lee, Appl. Phys. Lett. 103, 063501 (2013).

5. S. Guenneau, Y. Gao, and D. Veynante, Opt. Express 20, 8207 (2012).

6. J. Li, Y. Gao, and J. Huang, J. Appl. Phys. 108, 074504 (2012).

7. T. Yang, K. Vemuri, and P. Bandaru, Appl. Phys. Lett. 105, 083908 (2014). 
8. S. Narayana and Y. Sato, Phys. Rev. Lett. 108, 214303 (2012).

9. L. Zeng, Phys. Lett. A 378, 923 (2014).

10. J. Pendry, D. Shurig, and D. Smith, Science 312, 1780 (2006).

11. U. Leonhardt, Science 312, 1777 (2006).

12. G. Milton, M. Briane, and J. Willis, New J. Phys. 8, 248 (2006).

13. M. Moccia, G. Castaldi, S. Savo, Yu Sato, and V. Galdi, Phys. Rev. X 02, 2014 (1025).

14. L. Zeng, Z. Tang, H. Li, Y. Zhao, C. Dai, and R. Song, Modern. Phys. Lett. B 28, 1450098 (2014).

15. R. Schittny, M. Kadic, S. Guenneau, and M. Wegener, Phys. Rev. Lett. 110, 195901 (2013).

16. S. Narayana, S. Savo, and Y. Sato, Appl. Phys. Lett. 102, 201904 (2013).
17. T. Han, X. Bai, D. Gao, J. Thong, B. Li, and C. Qiu, Phys. Rev. Lett. 112, 054302 (2014).

18. T. Han, T. Yuan, B. Li, and C.W. Qiu, Sci. Rep. 3, 1593 (2013).

19. R.H. Tarkhanyan and D.G. Niarchos, Int. J. Therm. Sci. 67, 107 (2013).

20. Z. Tian, S. Lee, and G. Chen, J. Heat Transf. 135, 061605 (2013).

21. R.H. Tarkhanyan and D.G. Niarchos, J. Electron. Mater. 43, 3808 (2014).

22. Y.S. Touloukian, R.W. Powell, C.W. Ho, and P.G. Klemens, Thermophysical properties of matter, Vol. 1 (New York: IFI/ Plenum, 1970), p. 339.

23. L. Weber and E. Gmelin, J. Appl. Phys. A 53, 136 (1991).

24. J.M. Ziman, Electrons and phonons, (Chapt. 7) (Oxford: Oxford University Press, 1960), p. 286.

25. A.I. Ansel'm, Introduction to semiconductor theory (Moscow: Mir, 1981) 